\title{
INTEGRABILITY OF LIÉNARD SYSTEMS WITH A WEAK SADDLE
}

\author{
ARMENGOL GASULL ${ }^{1}$ AND JAUME GINÉ ${ }^{2}$
}

\begin{abstract}
We characterize the local analytic integrability of weak saddles for complex Liénard systems.
\end{abstract}

\section{INTRODUCTION AND MAIN RESUlTS}

Since the pioneering works of Élie and Henri Cartan ([1]) and Balthasar van der Pol $([23,24])$ where Liénard type differential equations([14]),

$$
\frac{d^{2} x}{d t^{2}}+f(x) \frac{d x}{d t}+g(x)=\ddot{x}+f(x) \dot{x}+g(x)=0,
$$

appear in electrical problems, many other situations have been modeled by differential equations that can be transformed into them. For instance, Liénard equations appear in mechanical problems, in predator-prey systems $([8])$ and chemical or biochemical reactions $([16,18])$. It is also remarkable that Hilbert 16th problem restricted to planar polynomial quadratic differential equations, which recall that in this case asks about a uniform upper bound for the number of limit cycles that the quadratic systems can have, can be reduced to the study of some Liénard type differential equations, see [25].

Two of the main questions about them are to know whether they are integrable or not, and to give criteria for controlling their number of limit cycles. This paper deals with the first one.

Poincaré proved that if a real planar analytic differential system has a weak focus at a critical point, then this point is a center if and only if the equation has an analytic first integral defined in a neighborhood of this point, see for instance $[13,17,19,21]$. Then the characterization of the centers for non-degenerate critical points is equivalent to the characterization of the local analytic integrable cases.

Let us recall the simple and well understood characterization of the centers, and so the local analytic integrable cases, for classical real analytic Liénard systems, $g(x) \equiv x$. If we write these equations in $\mathbb{R}^{2}$ as

$$
\dot{x}=\frac{d x}{d t}=y+F(x), \quad \dot{y}=-x,
$$

with $F(x)=-\int_{0}^{x} f(s) d s$ analytic at zero and $F(0)=F^{\prime}(0)=0$, then the origin is a center if and only if $F$ is an even function, see for instance $[3,6,27]$. We include for completeness a very simple proof, which essentially is the one appearing in $[3,27]$. Write $F=F^{o}+F^{e}$, where $F^{e}(x)=(F(x)+F(-x)) / 2$ is the even part of $F$ and $F^{o}$ its odd part. If $F=F^{e}$, then the origin is a center by the classical criterion of Poincaré: the origin is a monodromic critical point (a center or a focus) and the differential equation is reversible, because it is invariant by the change of variables and time $(x, y, t) \rightarrow(-x, y,-t)$. If $F \neq F^{e}$, then $F^{o}(x)=a x^{2 k+1}+O_{2 k+2}(x)$

2010 Mathematics Subject Classification. Primary 37C15. Secondary 34A34; .

Key words and phrases. Center problem; Analytic integrability, Weak saddle; Liénard equation. 
with $a \neq 0, k \in \mathbb{N}$ and we need to prove that the origin is not a center. Consider the scalar product

$$
(y+F(x),-x) \cdot\left(y+F^{e}(x),-x\right)^{\perp}=x\left(F(x)-F^{e}(x)\right)=x F^{o}(x)=a x^{2 k+2}+O_{2 k+3}(x),
$$

where, as usual $(u, v)^{\perp}=(-v, u)$ is a perpendicular vector to $(u, v)$. Then, on a neighborhood of the origin, it does not change sign and vanishes only on $x=0$, which is not an invariant curve of the Liénard system. Therefore, the level sets of the Liénard system corresponding to $F=F^{e}$, which are closed curves surrounding the origin, are curves without contact for the flow of the original Liénard system. This implies that the origin is not a center, as we wanted to prove. In other words, we have used the first integral of the integrable case $F=F^{e}$ as a Lyapunov function for the non-integrable one $F \neq F^{e}$. Another proof of this result is given in [5]. It is based on the computation of the so-called Lyapunov constants, that are the obstructions for finding an analytic first integral of the Liénard system.

One of the motivations of this paper is to prove a similar result, but for classical Liénard systems with a weak saddle at the origin,

$$
\dot{x}=y+F(x), \quad \dot{y}=x,
$$

with $F$, also analytic and $F(0)=F^{\prime}(0)=0$. Notice that its eigenvalues are \pm 1 . Recall that a saddle point is called weak if its eigenvalues are $\pm \lambda$ with $0 \neq \lambda \in \mathbb{R}$. That is, weak saddles are hyperbolic saddles such that the divergence of the vector field vanishes on them.

In this situation we have not the clear geometric interpretation of integrable system as the one for which the orbits are closed and a more analytical approach is needed. In fact, the method that we will use is the computation of the so-called (resonant) saddle quantities that we will recall below, which as we will see, is similar to the computation of the Lyapunov quantities used in [22]. It is also worth to mention that the duality between centers and saddles, given by the complex change of variables and time $\left(x_{1}, y_{1}, t_{1}\right)=(i x, y$, it $)$, that transforms one case into the other one when considering the system in $\mathbb{C}^{2}$, does not allow to use the simple geometric interpretation that we have on $\mathbb{R}^{2}$. Other Liénard-like complex systems with a weak saddle are studied in [10].

Before stating our main result, we introduce some notation and motivate the extension of our problem to $\mathbb{C}^{2}$.

The general center problem for analytic vector fields in $\mathbb{R}^{2}$ with an elementary singular point of the form

$$
\dot{x}=-y+O_{2}(x, y), \quad \dot{y}=x+O_{2}(x, y),
$$

is sometimes embedded by the change of variable $u=x+i y$ and the corresponding conjugate variable $v=x-i y$ into the complex vector fields of the form

$$
\dot{u}=u+O_{2}(u, v), \quad \dot{v}=-v+O_{2}(u, v) .
$$

The next extension of the above system is to consider analytic vector fields in $\mathbb{C}^{2}$ of the form

$$
\dot{u}=\lambda u+O_{2}(u, v), \quad \dot{v}=-\mu v+O_{2}(u, v),
$$

where $\lambda, \mu \in \mathbb{C} \backslash\{0\}$.

It is already proved by Poincaré $([12,20])$ that if $\lambda / \mu \notin \mathbb{Q}^{+}$then the differential equation (1) has no local analytic first integral in a neighborhood of zero. The case $\lambda / \mu=p / q \in \mathbb{Q}^{+}$, with $\operatorname{gcd}(p, q)=1$, is called $[p:-q]$ resonant case and in this situation, adding some more necessary conditions, the local analytic integrability is sometimes possible. Let us briefly recall how to obtain these conditions.

Changing the time, if necessary, the $[p:-q]$ resonant case can be written as

$$
\dot{u}=p u+O_{2}(u, v), \quad \dot{v}=-q v+O_{2}(u, v),
$$


with $p, q \in \mathbb{Z}^{+}$. For this system the linear part has the analytic first integral $H_{0}(u, v)=$ $u^{q} v^{p}$ and we can seek the conditions for the existence of an analytic first integral $H(u, v)=$ $H_{0}(u, v)+O_{p+q+1}(u, v)$ for system (2). Hence we get the equation $\dot{H}(u, v)=v_{1} H_{0}^{2}(u, v)+$ $v_{3} H_{0}^{3}(u, v)+\cdots$, and the so-called $[p:-q]$ resonant saddle quantities $v_{i}$ are polynomials of the coefficients of system (2). If all the $v_{i}$ are zero we say that we have a formal analytic resonant saddle, see $[11,26]$ and references therein. From this result we obtain also the existence of a local analytic first integral, see [21].

In this work we aim to give a simple and self-contained proof of the characterization of the integrable complex analytic differential systems in $\mathbb{C}^{2}$ of the form

$$
\dot{x}=y+F(x), \quad \dot{y}=a x, \quad 0 \neq a \in \mathbb{C},
$$

where $F(x)$ is an analytic function of $x$ without linear and constant terms. We prove:

Theorem 1. System (3) is locally integrable at the origin if and only if $F(x)$ is and even function of $x$.

Notice that if we restrict our attention to $(x, y) \in \mathbb{R}^{2}$, our result covers the classical Liénard case with a weak focus at the origin, $a=-1$, and the weak saddle case, $a=1$, which has a $[1:-1]$ resonance. Our result also extends to $\mathbb{C}^{2}$ some of the results of [15].

As a corollary of Theorem 1 and using the ideas of Cherkas([3]) and Christopher, Lloyd and Pearson $([6])$ we get the characterization of the real analytic integrable weak saddles for general Liénard systems, see also $[2,7,9]$. This corollary is also recently proved in [15] and extends to the weak saddle case the known results for the weak focus case.

Consider the differential systems in $\mathbb{R}^{2}$ of the form

$$
\dot{x}=y+F(x), \quad \dot{y}=g(x),
$$

where $F$ and $g$ are analytic functions of $x, F(x)$ without linear and constant terms and with $g(0)=0$ and $g^{\prime}(0) \neq 0$ and set $G(x)=\int_{0}^{x} g(\xi) d \xi$.

Corollary 2. System (4) has an integrable resonant weak saddle (resp. weak focus) at the origin if and only if $g^{\prime}(0)>0$ (resp. $\left.g^{\prime}(0)<0\right)$ and $F(x)=\phi(G(x))$, for some analytic function $\phi$ with $\phi(0)=0$.

\section{Proof of the Results}

Proof of Theorem 1. System (3) can be transformed into system

$$
\dot{x}=y+F(x), \quad \dot{y}=x,
$$

doing the change of variables $x=x, Y=b y$ and the change of time $d t=b d s$ with $b$ any of the roots $1 / \sqrt{a}$. Observe that for simplicity we write again $y$ instead of $Y$.

Consider $F(x)=\sum_{i=2}^{\infty} a_{i} x^{i}$. As explained in the introduction, to find the saddle quantities we propose a formal first integral of the form

$$
H(x, y)=x^{2}-y^{2}+\sum_{k=3}^{\infty} H_{k}(x, y),
$$

where $H_{k}(x, y)$ are homogeneous polynomials that can be written

$$
H_{k}(x, y)=\sum_{i+j=k} c_{i, j} x^{i} y^{j} .
$$


Now we compute the derivative of $H$ along the vector field associated to system (5) and we obtain a linear system for each function $H_{k}$. For instance for $H_{3}$ we have the linear system

$$
\left(\begin{array}{llll}
0 & 1 & 0 & 0 \\
3 & 0 & 2 & 0 \\
0 & 2 & 0 & 3 \\
0 & 0 & 1 & 0
\end{array}\right)\left(\begin{array}{c}
c_{3,0} \\
c_{2,1} \\
c_{1,2} \\
c_{0,3}
\end{array}\right)=-\left(\begin{array}{c}
2 a_{2} \\
0 \\
0 \\
0
\end{array}\right) .
$$

From the last equation we obtain $c_{1,2}=0$. Now we go to the second equation and we have $c_{3,0}=0$. These two conditions are the only ones that we will use for the next steps of the proof. The linear system for $H_{4}$ is

$$
\left(\begin{array}{lllll}
0 & 1 & 0 & 0 & 0 \\
4 & 0 & 2 & 0 & 0 \\
0 & 3 & 0 & 3 & 0 \\
0 & 0 & 2 & 0 & 4 \\
0 & 0 & 0 & 1 & 0
\end{array}\right)\left(\begin{array}{c}
c_{4,0} \\
c_{3,1} \\
c_{2,2} \\
c_{1,3} \\
c_{0,4}
\end{array}\right)=-\left(\begin{array}{c}
2 a_{3}+3 a_{2} c_{3,0} \\
2 a_{2} c_{2,1} \\
a_{2} c_{1,2} \\
0 \\
0
\end{array}\right)
$$

From the last equation we obtain $c_{1,3}=0$. Next, we consider the third equation, and taking into account that $c_{1,2}=0$, we deduce $c_{3,1}=0$. Finally, the first equation gives us that $a_{3}$ must be zero, taking into account that $c_{3,0}=0$, obtaining the first condition to have formal integrability for system (5). The linear system for $H_{5}$ is

$$
\left(\begin{array}{llllll}
0 & 1 & 0 & 0 & 0 & 0 \\
5 & 0 & 2 & 0 & 0 & 0 \\
0 & 4 & 0 & 3 & 0 & 0 \\
0 & 0 & 3 & 0 & 4 & 0 \\
0 & 0 & 0 & 2 & 0 & 5 \\
0 & 0 & 0 & 0 & 1 & 0
\end{array}\right)\left(\begin{array}{c}
c_{5,0} \\
c_{4,1} \\
c_{3,2} \\
c_{2,3} \\
c_{1,4} \\
c_{0,5}
\end{array}\right)=-\left(\begin{array}{c}
2 a_{4}+3 a_{3} c_{3,0}+4 a_{2} c_{4,0} \\
2 a_{3} c_{2,1}+3 a_{2} c_{3,1} \\
a_{3} c_{1,2}+2 a_{2} c_{2,2} \\
a_{2} c_{1,3} \\
0 \\
0
\end{array}\right)
$$

From the last equation we have $c_{1,4}=0$, now going to the fourth one, and taking into account that $c_{1,3}=0$, we arrive to $c_{3,2}=0$. Finally, from the second equation, and taking into account that $a_{3}=0$ and $c_{3,1}=0$, we have $c_{5,0}=0$. Similarly, the linear system for $H_{6}$ is

$$
\left(\begin{array}{ccccccc}
0 & 1 & 0 & 0 & 0 & 0 & 0 \\
6 & 0 & 2 & 0 & 0 & 0 & 0 \\
0 & 5 & 0 & 3 & 0 & 0 & 0 \\
0 & 0 & 4 & 0 & 4 & 0 & 0 \\
0 & 0 & 0 & 3 & 0 & 5 & 0 \\
0 & 0 & 0 & 0 & 2 & 0 & 6 \\
0 & 0 & 0 & 0 & 0 & 1 & 0
\end{array}\right)\left(\begin{array}{c}
c_{6,0} \\
c_{5,1} \\
c_{4,2} \\
c_{3,3} \\
c_{2,4} \\
c_{1,5} \\
c_{06}
\end{array}\right)=-\left(\begin{array}{c}
2 a_{5}+3 a_{4} c_{3,0}+4 a_{3} c_{4,0}+5 a_{2} c_{5,0} \\
2 a_{4} c_{2,1}+3 a_{3} c_{3,1}+4 a_{2} c_{4,1} \\
a_{4} c_{1,2}+2 a_{3} c_{2,2}+3 a_{2} c_{3,2} \\
a_{3} c_{1,3}+2 a_{2} c_{2,3} \\
a_{2} c_{1,4} \\
0 \\
0
\end{array}\right) .
$$

Now, taking into account that $c_{1,2}=c_{3,0}=0, c_{1,4}=c_{3,2}=c_{5,0}=0$, and $a_{3}=0$ we obtain that $c_{1,5}=c_{3,3}=c_{5,1}=0$ and that $a_{5}$ must be zero, that is the second condition to have integrability for system (5).

Let us prove the theorem by induction. Our induction hypothesis is that for each $n$ odd, the necessary conditions for system (5) to have a formal first integral $H$ of the form (6) are:

$$
c_{n, 0}=c_{n-2,2}=\cdots=c_{3, n-3}=c_{1, n-1}=0, \quad c_{n, 1}=c_{n-2,3}=\cdots=c_{3, n-2}=c_{1, n}=0,
$$

and $a_{3}=a_{5}=\cdots=a_{n}=0$.

In fact, until now, we have prove the induction hypothesis for $n=3$ and $n=5$. Clearly, the proof for $n=5$ is not needed to use the induction method but we have included it for the sake of clarity. 
For any $m$ we have that the linear system obtained that (6) is a formal first integral is

$$
\begin{gathered}
\left(\begin{array}{ccccccc}
0 & 1 & 0 & 0 & 0 & \cdots & 0 \\
m & 0 & 2 & 0 & 0 & \cdots & 0 \\
0 & m-1 & 0 & 3 & 0 & \cdots & 0 \\
0 & \cdots & \ddots & 0 & \ddots & \cdots & 0 \\
0 & \cdots & 0 & 3 & 0 & m-1 & 0 \\
0 & \cdots & 0 & 0 & 2 & 0 & m \\
0 & \cdots & 0 & 0 & 0 & 1 & 0
\end{array}\right)\left(\begin{array}{l}
c_{m, 0} \\
c_{m-1,1} \\
c_{m-2,2} \\
\vdots \\
c_{2, m-2} \\
c_{1, m-1} \\
c_{0, m}
\end{array}\right)= \\
\\
\\
\quad\left(\begin{array}{c}
2 a_{m-1}+3 a_{m-2} c_{3,0}+\cdots+(m-2) a_{3} c_{m-2,0}+(m-1) a_{2} c_{m-1,0} \\
2 a_{m-2} c_{21}+3 a_{m-3} c_{31}+\cdots+(m-3) a_{3} c_{m-3,1}+(m-2) a_{2} c_{m-2,1} \\
a_{m-3} c_{12}+2 a_{m-4} c_{22}+\cdots+(m-4) a_{3} c_{m-4,2}+(m-3) a_{2} c_{m-3,2} \\
\vdots \\
a_{2} c_{1, m-2} \\
0 \\
0
\end{array}\right) .
\end{gathered}
$$

Let us prove our induction hypothesis for any $n$ (odd) assuming that it is true for $n-2$.

When $m=n$ is odd the determinant of the above linear system is different from zero and then the system is compatible and determined. Moreover, using the induction hypothesis and starting from the last equation and going up in the other equations we arrive to $c_{n, 0}=$ $c_{n-2,2}=\cdots=c_{3, n-3}=c_{1, n-1}=0$, as we wanted to prove.

However for $m=n+1$ even we have that the determinant of the corresponding linear system is zero. Anyhow, using again our induction hypothesis, from the last equation we have $c_{1, n}=0$ and going up in the equations we get that $c_{n, 1}=c_{n-2,3}=\cdots=c_{1, n}=0$ and the first equation reduces to

$$
0=2 a_{n}+3 a_{n-1} c_{3,0}+\cdots+(n-1) a_{3} c_{n-1,0}+n a_{2} c_{n, 0} .
$$

Then, we know that $c_{i, 0}=0$ if $i$ is odd and if $i$ is even the $c_{i, 0}$ is multiplied by $a_{i}$ with $i$ odd. Since, moreover again by induction hypothesis, we know that $a_{3}=a_{5}=\cdots=a_{n-2}=0$ we obtain $0=2 a_{n}$ which implies $a_{n}=0$, as we wanted to show.

This proves that system (5) has only a formal first integral when $F$ is even. Then, applying [21, Cor. 3.2.6] we have that under this hypothesis system (5) has an analytic first integral around the origin.

Proof of Corollary 2. Following [3, 6], let us see that system (4) can be locally transformed into a classical Liénard system in $\mathbb{R}^{2}$ of the form

$$
\dot{x}=y+\widetilde{F}(x), \quad \dot{y}=\sigma x,
$$

where $\sigma=\operatorname{sign}\left(g^{\prime}(0)\right)$ and $\widetilde{F}$ is an analytic function. Let $u$ be the root of $2 \sigma G(x)$ that has the same sign that $x$,

$$
u=\phi(x)=\operatorname{sign}(x) \sqrt{2 \sigma G(x)}=x \sqrt{\left|g^{\prime}(0)\right|\left(1+O_{1}(x)\right)},
$$

which is well defined and analytic in a neighborhood of $x=0$. Moreover, it has an analytic inverse $x=\xi(u)=u\left(1+O_{1}(u)\right) / \sqrt{\left|g^{\prime}(0)\right|}$. The transformation $u=\phi(x)$ converts system (4) into the new system

$$
\dot{u}=\frac{\sigma g(\xi(u))}{u}(y+F(\xi(u))), \quad \dot{x}=g(\xi(u))
$$


Since $g(\xi(u)) / u=\sigma \sqrt{\left.\mid g^{\prime}(0)\right) \mid}+O_{1}(u)$ is analytic and nonzero in a neighborhood of the origin we can do the change of time $d t / d s=\sigma u / g(\xi(u))$ and it becomes the classical Liénard system,

$$
u^{\prime}=y+F(\xi(u)), \quad \dot{x}=\sigma u .
$$

By Theorem 1 the condition to have integrability for such system is that $F(\xi(u))$ is an analytic even function of $u$. The chain of equalities

$$
F(\xi(u))=\widehat{\phi}\left(u^{2}\right)=\widehat{\phi}(2 \sigma G(x))=\phi(G(x))
$$

proves the corollary.

By using Lüroth theorem as in [4] we get the following effective characterization of real integrable polynomial Liénard systems with a weak saddle at the origin:

Corollary 3. Consider system (4) with $F$ and $G$ polynomials. Then it has an integrable resonant weak saddle at the origin if and only if there exist polynomials $\widehat{F}, \widehat{G}$ and $u$, with $u(0)=u^{\prime}(0)=0, u^{\prime \prime}(0) \neq 0$, such that

$$
F(x)=\widehat{F}(u(x)), \quad G(x)=\widehat{G}(u(x)) .
$$

The strong saddles case. The problem of the local integrability for strong (that is, nonweak) saddles of Liénard systems is not considered in our work. Notice that if instead of system (4) we take in $\mathbb{R}^{2}$ the system

$$
\dot{x}=y+F(x), \quad \dot{y}=x,
$$

with $F(0)=0$ but $F^{\prime}(0) \neq 0$, it has a strong saddle at the origin. It is convenient to write $F^{\prime}(0)=1 / c-c$, with $c \in \mathbb{R}^{+} \backslash\{1\}$. We remove the case $c=1$ to avoid the weak saddle case. Then its eigenvalues are $-c<0<1 / c$, and the Poincaré necessary condition for having an analytic first integral at the origin is $c^{2}=q / p \in \mathbb{Q}^{+}$. In fact, if $F(x) \equiv(\sqrt{p / q}-\sqrt{q / p}) x$ it is not difficult to prove that

$$
H(x, y)=(\sqrt{p} x+\sqrt{q} y)^{q}(\sqrt{p} y-\sqrt{q} x)^{p}
$$

is a first integral of the linear Liénard system. We do not know if there are nonlinear integrable cases in system (7).

\section{ACKNowledgements}

The first author is supported by a MINECO grant number MTM2013-40998-P and by a CIRIT grant number 2014SGR568. The second author is partially supported by a MINECO/ FEDER grant number MTM2014-53703-P and an AGAUR (Generalitat de Catalunya) grant number 2014SGR 1204.

\section{REFERENCES}

[1] É. Cartan, H. Cartan, Note sur la génération des oscillations entretenues, Annales des P.T.T. 14 (1925), 1196-1207.

[2] L.A. Cherkas, On the conditions for a center for certain equations of the form yy' $=P(x)+Q(x) y+$ $R(x) y^{2}$, Differ. Uravn. 8 (1972), 1435-1439; Differ. Equ. 8 (1972), 1104-1107.

[3] L.A. Cherkas, Conditions for a Liénard equation to have a centre, Differ. Equ. 12 (1976), $201-206$.

[4] C.J. Christopher, An algebraic approach to the classification of centres in polynomial Liénard systems, J. Math. Anal. Appl. 229 (1999), 319-329.

[5] C. Christopher, N.G. Lloyd, Small-amplitude limit cycles in polynomial Liénard systems, NoDEA 3 (1996), 183-190.

[6] C.J. Christopher, N.G. Lloyd, J.M. Pearson, On a Cherkass method for centre conditions, Nonlinear World 2 (1995), 459-469. 
[7] A. Gasull, J. Torregrosa, Center problem for several differential equations via Cherkas' method, J. Math. Anal. Appl. 228 (1998), 322-343.

[8] G.F. Gause, The struggle for existence, Baltimore, Williams and Wilkins, 1934; new edition New York, Dover, 1971.

[9] J. Giné, Center conditions for polynomial Liénard systems, preprint. Universitat de Lleida, 2015.

[10] J. Giné, C. VALLS, Integrability conditions of a resonant saddle in Liénard-like complex systems, Chaos Solitons Fractals 82 (2016), 139-141.

[11] A. Fronville, A. Sadovski, H. Zoladek, Solution of the $1:-2$ resonant center problem in the quadratic case. Dedicated to the memory of Wieslaw Szlenk. Fund. Math. 157 (1998), 191-207.

[12] S.D. FurTA, On non-integrability of general systems of differential equations, Z. angew Math. Phys. 47 (1996), 112-131.

[13] A.M. Liapunov, Stability of Motion, Mathematics in Science and Engineering, Vol. 30 Academic Press, New York-London, 1966.

[14] A. LiÉNARD, Etude des oscillations entretenues, Revue géner. de l'electr. 23 (1928), 901-906; 906-954.

[15] J. Llibre, C. VAlLs, On the analytic integrability of the Liénard analytic differential systems, Disc. Cont. Dyn. Sys. 21 (2016) 557-573.

[16] H. MaOAn, On limit cycles of a biochemical reaction system, J. Nanjing Univ., Math. Biquart. (1986), $50-53$.

[17] R. Moussu, Une démonstration d'un théorème de Lyapunov-Poincaré, Astérisque 98/99 (1982), 216223.

[18] G. Nicolis, I. Prigogine, Self-Organization in nonequilibrium system, Wiley, New York, 1977.

[19] H. Poincaré, Mémoire sur les courbes définies par les équations differentielles, Journal de Mathématiques, 37 (1881), 375-422; Oeuvres de Henri Poincaré, vol. I, Gauthier-Villars, Paris, 1951, pp $3-84$.

[20] H. Poincaré, Sur l'intégration des équations différentielles du premier order et du premier degré I and II, Rendiconti del circolo matematico di Palermo, 5 (1891), 161-191; 11 (1897), 193-239.

[21] V.G. Romanovski, D.S. Shafer, The Center and Cyclicity Problems: A Computational Algebra Approach, Birkhäuser, Boston, 2009.

[22] S.L. SHI, A method of constructing cycles without contact around a weak focus. J. Differential Equations 41 (1981), 301-312.

[23] B. VAN Der Pol, Sur les oscillations de relaxation, Philosophical Magazine 2 (1926), 978-992.

[24] B. VAn DER Pol, The non linear theory of electrical oscillations, Proc. Inst. Radio Eng. 22 (1934), $1051-1086$.

[25] Y. YAN-QIAN and others, Theory of limit cycles. Translations of Mathematical Monographs, AMS vol. 66, Rhode Island, 1986.

[26] Q. Zhang, Y. LiU, Integrability and generalized center problem of resonant singular point Appl. Math. Lett. 40 (2015), 13-16.

[27] C. Zuppa, Order of cyclicity of the singular point of Liénard's polynomial vector fields, Bol. Soc. Brasil Mat. 12 (1982), 105-111.

1 Departament de Matemàtiques, Universitat Autònoma de Barcelona, 08193 Bellaterra, Barcelona, Catalonia, Spain

E-mail address: gasull@mat.uab.cat

2 Departament de Matemàtica, Universitat de Lleida, Avda. Jaume II, 69; 25001 Lleida, Catalonia, Spain

E-mail address: gine@matematica.udl.cat 